Enferm Bras 2020;19(4);345-54

https://doi.org/10.33233/eb.v19i4.4281

\title{
REVISÃo \\ Sexualidade de idosas e contribuições da enfermagem
}

Valquiria Maria de Paula, M.Sc. ${ }^{*}$, Leiner Resende Rodrigues, D.Sc. **

*Universidade Federal do Triângulo Mineiro (UFTM), **Profa Associada do Departamento de Enfermagem em Educação e Saúde Comunitária, Universidade Federal do Triângulo Mineiro

Recebido em 17 de julho de 2020; aceito em 30 de agosto de 2020.

Correspondência: Valquiria Maria de Paula, Rua Bernardo Rossi, 1450 Bom Retiro, 38022210 Uberaba MG

Valquiria Maria de Paula: valquiriacig@yahoo.com.br

Leiner Resende Rodrigues: leiner.r.rodrigues@gmail.com

\section{Resumo}

Introdução: A sexualidade de idosas é um tema delicado, repleto de tabus e de dificuldade global. Há necessidade da enfermagem em construir práticas em saúde abordando melhor o assunto, em busca de novas concepções e avanços na área. Objetivo: Conhecer as contribuições da enfermagem para sexualidade de idosas. Métodos: Revisão narrativa realizada nas bases de dados Biblioteca Virtual em Saúde, Pubmed e Scientific Electronic Library Online, abordando estudos de 2016 a 2020, em português, inglês e espanhol, utilizando os descritores cuidados de enfermagem, sexualidade e mulheres idosas. Procedeu-se a leitura e análise das publicações. Resultados: Poucos estudos foram encontrados especificando idosas e cuidados de enfermagem. Dentre as contribuições de enfermagem elencadas, temos: educação em saúde para idosas sobre sexualidade, capacitação da equipe de enfermagem, pesquisas na área, apoio e acolhimento, consulta de enfermagem, coleta de Papanicolau, sensibilização da enfermagem e das idosas sobre saúde sexual. No entanto, a mais evidenciada foi educação em saúde. Conclusão: A enfermagem pode contribuir de vários modos e nos diversos espaços do cuidar, em busca do cuidado holístico, vida sexual saudável e satisfatória, livre de preconceitos e violência.

Palavras-chave: cuidados de enfermagem, mulheres idosas, sexualidade.

\section{Abstract \\ Sexuality of elderly women and nursing contributions}

Introduction: The sexuality of elderly women is a delicate topic, full of taboos and global difficulties. There is a need for nursing to build health practices by better addressing the subject, in search of new conceptions and advances in the area. Objective: To know the nursing contributions to the sexuality of elderly women. Methods: Narrative review carried out in the Virtual Health Library, Pubmed and Scientific Electronic Library Online databases, addressing studies from 2016 to 2020, in Portuguese, English and Spanish, using the keywords nursing care, sexuality and elderly women. The publications were read and analyzed. Results: Few studies were found specifying elderly women and nursing care. Among the nursing contributions listed, we have: health education for elderly women about sexuality, training of the nursing team, research in the area, support and reception, nursing consultation, Pap smear collection, nursing and elderly awareness about sexual health. However, the most evident was health education. Conclusion: Nursing can contribute in various ways and in different spaces of care, in search of holistic care, healthy and satisfactory sexual life, free of prejudice and violence.

Keywords: nursing care, elderly women, sexuality.

\section{Resumen}

Sexualidad de las mujeres mayores y contribuciones de la enfermería 
Introducción: La sexualidad de las mujeres mayores es un tema delicado, lleno de tabúes y dificultades globales. Es necesario que la enfermería construya prácticas de salud mediante un mejor enfoque del tema, en busca de nuevas concepciones y avances en el área. Objetivo: Conocer las contribuciones de la enfermería a la sexualidad de las mujeres mayores. Métodos: Revisión narrativa realizada en las bases de datos de la Biblioteca Virtual en Salud, Pubmed y Scientific Electronic Library Online, abordando estudios de 2016 a 2020, en portugués, inglés y español, utilizando los descriptores atención de enfermería, sexualidad y mujeres mayores. Las publicaciones fueron leídas y analizadas. Resultados: Se encontraron pocos estudios que especificaran mujeres mayores y atención de enfermería. Entre las contribuciones de enfermería listadas, tenemos: educación en salud para las mujeres mayores sobre la sexualidad, formación del equipo de enfermería, investigación en el área, apoyo y acogida, consulta de enfermería, recogida de la prueba de Papanicolaou, sensibilización de la enfermería y de las mujeres mayores sobre la salud sexual. Sin embargo, lo más evidente fue la educación en salud. Conclusión: La enfermería puede contribuir de varios modos y en los varios espacios de atención, en busca de una atención holística, una vida sexual sana y satisfactoria, libre de prejuicios y violencia.

Palabras-clave: atención de enfermería, mujeres mayores, sexualidad.

Introdução

A sexualidade é entendida numa perspectiva ampla, não se limitando ao ato sexual, pode ser expressa através do abraço, do carinho e do companheirismo, é uma manifestação presente em todas as fases da vida [1]. Vem sendo investigada com maior ênfase desde o século XIX. A sexualidade de idosas é um tema delicado, repleto de tabus e de dificuldade global. Fatores psicológicos, socioculturais e biológicos apresentam repercussão para a prática sexual saudável dessa população $[2,3]$.

A forma como as mulheres vivenciam e percebem sua sexualidade representa a ligação entre o conhecimento científico e o senso comum, trazendo informações para a saúde sexual e o bem-estar [4]. As idosas de hoje tiveram uma educação rígida estabelecida desde os séculos anteriores, seguindo a cultura da preservação da virgindade até o casamento, papéis voltados para serviços domésticos, reprodução e criação de filhos e submissão ao cônjuge $[5,6]$. Historicamente sua sexualidade foi reprimida e silenciada, considerada como algo impuro, exclusivo à reprodução e não ao prazer $[1,3]$.

A idosa sente medo de tornar-se ridícula, por isso, mantém uma postura mais discreta. Seus familiares não estimulam novos relacionamentos amorosos, desta forma, ela tem uma relação de submissão aos filhos e presas aos julgamentos dos mesmos [5,7]. No entanto, atualmente a mulher vem conquistando seu espaço e com o processo da feminização do envelhecimento, elas estão valorizando a vivência da sexualidade, buscando relações mais livres e com maior satisfação [8]. A ideia de apenas satisfazer o parceiro está mudando, mas, a afetividade tem papel fundamental na relação [4]. A prática sexual e o desejo não se esgotam com o envelhecimento, apenas se modificam, desmistificando a visão que a pessoa idosa é assexuada $[2,9]$.

Apesar de avanços, observamos a falta de capacitação de profissionais de saúde, sobretudo da enfermagem, para atender essa demanda livre de críticas e preconceitos, proporcionando melhora da qualidade de vida, cuidado integral e emancipador em saúde [9]. A abordagem inadequada dos profissionais, considerando que os idosos não têm vida sexual ativa atua negativamente, pois eles não dialogam e nem questionam sobre a prática sexual, interferindo na qualidade da assistência, tornando um problema vivenciado, mas não tratado e negligenciando essa necessidade humana básica $[3,10]$.

É imprescindível aprofundarmos nessa temática, pois vem ocorrendo uma revolução sexual, refletindo nas idosas, dessa forma, concretizaremos os benefícios da sexualidade saudável. Nesta perspectiva, há uma necessidade da enfermagem em construir práticas em saúde abordando melhor a sexualidade no envelhecimento, em busca de novas concepções e propostas de estudos para avanços na área [11]. Considerando que pesquisas nesse assunto são escassas e que existem lacunas na literatura, o objetivo deste estudo foi conhecer as contribuições da enfermagem para a sexualidade de idosas. 
Trata-se de uma revisão narrativa da literatura. Os artigos de revisão narrativa são publicações amplas que descrevem um determinado assunto, do ponto de vista teórico, utilizando publicações de livros e revistas impressas e/ou eletrônicas. Permite ao leitor atualização do conhecimento sobre um tema em curto espaço de tempo [12].

Foram realizadas buscas nas bases de dados BVS (Biblioteca Virtual em Saúde), Pubmed e Scielo (Scientific Electronic Library Online), com os Descritores em Ciências da Saúde (DeCS) e Medical Subject Headings (MeSH): Cuidados de enfermagem (Nursing care); Sexualidade (Sexuality); Mulheres idosas (Elderly women). Critérios de inclusão dos artigos: pesquisas disponíveis na íntegra, em português, inglês e espanhol, publicadas no período de 2016 a 2020. Como exclusão consideramos os trabalhos não disponíveis na íntegra, repetidos e aqueles que não responderam ao objetivo da revisão.

Nas bases de dados utilizadas e com os descritores citados acima, encontramos: 9 publicações na BVS; 0 na Pubmed; 0 na Scielo. No entanto, para o estudo foram usadas apenas 7 da BVS. Procedeu-se a leitura detalhada das publicações e realizada a interpretação e discussão dos resultados.

Os aspectos éticos deste trabalho foram preservados. Todos os autores dos artigos utilizados foram referenciados adequadamente. As informações apresentadas são fidedignas.

\section{Resultados}

A combinação dos descritores e a aplicação de critérios de inclusão e exclusão resultou em 7 publicações na BVS. Na Pubmed e Scielo não foram encontradas publicações sobre 0 assunto proposto. A maioria das pesquisas engloba idosos de forma geral, poucos estudos foram encontrados especificando sexualidade de idosas e cuidados de enfermagem.

Para caracterização, análise e síntese das publicações inclusas, foi elaborado um quadro desenvolvido no programa Microsoft Word (Quadro 1), especificando de cada artigo: título, ano, tipo de estudo, objetivos e contribuições da enfermagem. 
Quadro 1- Caracterização, análise e síntese de artigos inclusos.

\begin{tabular}{|c|c|c|c|}
\hline $\begin{array}{l}\text { Titulo / } \\
\text { ano de publicação }\end{array}$ & Tipo de estudo & Objetivos & $\begin{array}{l}\text { Contribuiçőes da } \\
\text { enfermagem }\end{array}$ \\
\hline $\begin{array}{l}\text {-Vivência da } \\
\text { sexualidade por } \\
\text { mulheres idosas [13]. } \\
-2017\end{array}$ & $\begin{array}{l}\text {-Estudo } \\
\text { qualitativo/Teoria } \\
\text { fundamentada nos } \\
\text { dados. }\end{array}$ & $\begin{array}{l}\text {-Interpretar a vivência } \\
\text { da sexualidade pela } \\
\text { mulher idosa e } \\
\text { construir um modelo } \\
\text { teórico explicativo. }\end{array}$ & $\begin{array}{l}\text {-Capacitação da equipe } \\
\text { de enfermagem sobre } \\
\text { sexualidade de idosas. } \\
\text {-Pesquisas nessa } \\
\text { temática. }\end{array}$ \\
\hline $\begin{array}{l}\text { Sentidos de } \\
\text { sexualidade entre } \\
\text { mulheres idosas: } \\
\text { relaçôes de gênero, } \\
\text { ideologias } \\
\text { mecanicistas e } \\
\text { subversão [1]. } \\
-2018\end{array}$ & $\begin{array}{l}\text {-Estudo qual itativo de } \\
\text { caráter descritivo- } \\
\text { exploratório. }\end{array}$ & $\begin{array}{l}\text { - Compreender os } \\
\text { sentidos da } \\
\text { sexualidade } \\
\text { construidos por } \\
\text { mulheres idosas. }\end{array}$ & $\begin{array}{l}\text {-Educação em saúde } \\
\text { para idosas sobre } \\
\text { sexualidade. }\end{array}$ \\
\hline $\begin{array}{l}\text {-Concepção de } \\
\text { mulheres idosas } \\
\text { sobre a sexualidade } \\
\text { na velhice [8]. } \\
-2019\end{array}$ & $\begin{array}{l}\text {-Estudo qual itativo e } \\
\text { descritivo. }\end{array}$ & $\begin{array}{l}\text {-Analisar a } \\
\text { concepçẫo de } \\
\text { mulheres idosas } \\
\text { sobre a sexualidade } \\
\text { na velhice. }\end{array}$ & $\begin{array}{l}\text {-Educação em saúde } \\
\text { para idosas sobre } \\
\text { sexualidade, } \\
\text { sensibilizaçắo e } \\
\text { consultas de } \\
\text { enfermagem. }\end{array}$ \\
\hline $\begin{array}{l}\text {-Desvelamento crítico } \\
\text { em sexualidade entre } \\
\text { idosas como } \\
\text { dispositivo de } \\
\text { avaliação educativa } \\
\text { dialógica [14]. } \\
\text {-2019 }\end{array}$ & $\begin{array}{l}\text {-Estudo avaliativo, de } \\
\text { abordagem } \\
\text { qualitativa } \mathrm{e} \\
\text { construtivista. }\end{array}$ & $\begin{array}{l}\text {-Analisar as } \\
\text { percepç̃es sobre } \\
\text { sexualidade e os } \\
\text { respectivos } \\
\text { desvelamentos } \\
\text { críticos apreendidos } \\
\text { nos Círculos de } \\
\text { Cultura } \\
\text { desenvolvidos com } \\
\text { mulheres idosas. }\end{array}$ & $\begin{array}{l}\text {-Educaçăo em saúde } \\
\text { para idosas sobre } \\
\text { sexualidade. } \\
\text {-Pesquisas nessa } \\
\text { temática. }\end{array}$ \\
\hline $\begin{array}{l}\text {-0 percurso } \\
\text { educativo dialógico } \\
\text { como estratégia de } \\
\text { cuidado em } \\
\text { sexualidade com } \\
\text { idosas [15]. } \\
-2018\end{array}$ & $\begin{array}{l}\text {-Estudo qualitativo e } \\
\text { participativo, } \\
\text { abordagem de } \\
\text { pesquisa-ação } \\
\text { educativa, ancorado } \\
\text { no Itinerário de } \\
\text { Pesquisa de Freire. }\end{array}$ & $\begin{array}{l}\text {-Desvelar o } \\
\text { conhecimento crítico } \\
\text { mediado por um } \\
\text { percurso cuidativo- } \\
\text { educativo dialógico } \\
\text { em sexualidade com } \\
\text { mulheres idosas. }\end{array}$ & $\begin{array}{l}\text {-Educação em saúde } \\
\text { para idosas sobre } \\
\text { sexualidade. } \\
\text {-Educação/Capacitação } \\
\text { da equipe de } \\
\text { en fermagem sobre o } \\
\text { assunto. }\end{array}$ \\
\hline $\begin{array}{l}\text {-E nvelhecimento, } \\
\text { sexualidade e } \\
\text { cuidados de } \\
\text { en fermagem: o olhar } \\
\text { da mulher idosa [9]. } \\
-2019\end{array}$ & $\begin{array}{l}\text {-Estudo qual itativo e } \\
\text { descrivivo. }\end{array}$ & $\begin{array}{l}\text {-Analisar a percepção } \\
\text { da mulher idosa } \\
\text { sobre sexualidade e a } \\
\text { prática do cuidado de } \\
\text { enfermagem nesse } \\
\text { contexto. }\end{array}$ & $\begin{array}{l}\text {-Educação em } \\
\text { saúde/capacitação e } \\
\text { sensibilização para } \\
\text { idosas e profissionais } \\
\text { sobre sexualidade. } \\
\text {-Consultas de } \\
\text { en fermagem/colet a de } \\
\text { Papanicolau/acolhim ento. }\end{array}$ \\
\hline $\begin{array}{l}\text {-Repercussões da } \\
\text { menopausa para a } \\
\text { sexualidade de } \\
\text { idosas: revisão } \\
\text { integrativa da } \\
\text { literatura [16]. } \\
-2017\end{array}$ & -Revisão integrativa. & $\begin{array}{l}\text {-Apresentar uma } \\
\text { revisão integrativa da } \\
\text { literatura científica } \\
\text { nacional e } \\
\text { intemacional sobre as } \\
\text { possiveis } \\
\text { repercussões da } \\
\text { menopausa para a } \\
\text { sexualidade de } \\
\text { idosas. }\end{array}$ & $\begin{array}{l}\text {-Educação em } \\
\text { saúdelcapacitação e } \\
\text { sensibilização para } \\
\text { idosas e profissionais } \\
\text { sobre sexualidade. } \\
\text {-Pesquisas e apoio } \\
\text { emocional. }\end{array}$ \\
\hline
\end{tabular}

Discussão

Sete artigos compuseram a amostra desta revisão, a qual se propôs conhecer as contribuições da enfermagem para a sexualidade de idosas. Desses, seis utilizaram a metodologia qualitativa e um a revisão integrativa.

Dentre as contribuições de enfermagem elencadas, temos: educação em saúde para idosas sobre sexualidade, capacitação da equipe de enfermagem, pesquisas na área, apoio e acolhimento, consulta de enfermagem, coleta de Papanicolau, sensibilização da enfermagem e 
das idosas sobre saúde sexual. No entanto, a contribuição mais evidenciada foi educação em saúde.

\section{Educação em saúde com enfoque da sexualidade}

As práticas educativas são importantes ferramentas do cuidado, podendo ser realizadas em hospitais, Estratégia Saúde da Família, ambulatórios e instituições de longa permanência, seja, individualmente na consulta de enfermagem ou em formas de grupos $[17,18]$.

A educação em saúde possibilita o empoderamento social, influenciando na qualidade de vida, deve atuar na perspectiva de promoção da saúde com uma visão positiva da sexualidade no envelhecimento [14,15]. $\mathrm{Na}$ atualidade, a metodologia ativa de ensino vem sendo utilizada por profissionais da saúde para intervenções na população. Esse modelo é uma superação do ensino tradicional e passivo, incorpora vivências e realidade dos participantes através do diálogo e reflexão crítica, transformando saberes e práticas em busca da autonomia e emancipação dos educandos, eles serão protagonistas do seu aprendizado. Esse método é baseado no pensamento freiriano proporcionando ação-reflexão-ação, com dimensão coletiva e social [19].

O Itinerário de Pesquisa de Freire é composto pelas seguintes etapas: investigação temática (entrevistas e círculos de cultura); codificação (categorização das situações-limites e definição dos temas geradores); decodificação (dos temas geradores em conteúdo programático organizado em círculos de cultura); desvelamento crítico (em círculos de cultura) [19].

O círculo de cultura é formado por um grupo de pessoas que se reúne para discutir sua realidade, tendo como propósito analisar a prática cotidiana e atuar provocando mudanças de atitude e melhoria da realidade vivenciada. Temas geradores são extraídos da experiência de vida dos participantes sobre os problemas pensados a partir de "situações-limite" vivenciadas no dia-a-dia. Codificação/decodificação representa o momento de contextualização quando os temas são problematizados, questionados, analisados e os participantes começam a ter visão crítica do assunto discutido. Desvelamento crítico é o processo de ação-reflexão-ação para a superação das contradições da realidade vivida. É a fase da tomada de consciência da situação real [19].

Nos círculos de cultura as participantes são alocadas em cadeiras dispostas em círculos para melhor comunicação. O pesquisador é considerado como mobilizador, mediador, ou moderador da ação-reflexão-ação, realizada pelos participantes, mantendo relação horizontal e liberdade para o diálogo [19].

A enfermagem pode utilizar dessa ferramenta promissora para abordagem de temas como: sexualidade na terceira idade (concepções, vivência e satisfação), prevenção de doenças sexualmente transmissíveis e Síndrome da Imunodeficiência Adquirida (AIDS), disfunções sexuais, exames de mama e Papanicolau.

A educação em saúde contribui para a saúde integral, ultrapassando o processo saúdedoença, possibilitando a promoção em saúde e humanização [15].

\section{Capacitação da equipe de enfermagem: um novo olhar}

A sexualidade da idosa é permeado de discriminação, rejeição e mal-entendido, interferindo na assistência de enfermagem, restringindo ações e eliminando o tema da atuação do enfermeiro [9].

Profissionais da saúde têm dificuldades de abordagem desse assunto, preocupam-se com doenças crônicas, isso se deve ao despreparo e da valorização do corpo jovem, um reforço para o preconceito da sexualidade no envelhecimento $[9,16]$. Dessa forma, torna-se imprescindível a capacitação para profissionais, em especial da enfermagem, que é a categoria que tem mais contato com a cliente [2,20]. A formação acadêmica e a educação continuada apresentam lacunas, impedindo o cuidado integral [21].

A atuação sobre aspectos da sexualidade desvela condutas que se diversificam entre os profissionais e suas subjetividades, não há padronizações ou recomendações institucionais [21].

A manutenção de capacitação dos profissionais envolvidos deve ser contínua, contribuindo para assistência qualificada. As metodologias ativas de aprendizagem podem ser utilizadas pelo enfermeiro para treinar sua equipe e outros enfermeiros sobre sexualidade, despertando interesse e propondo prática pedagógica ética, crítica, reflexiva e transformadora [22].

Os enfermeiros devem estar preparados para acompanhar as idosas nessa transição, partilhando conhecimentos e evidências científicas para o envelhecimento ativo [9]. 
$\mathrm{Na}$ área de saúde, a maioria das produções científicas trata a sexualidade voltada para questões biológicas [13]. Por muito tempo esse tema foi deixado de lado e negligenciado na área de pesquisa, devido ao desinteresse de profissionais e a inibição de idosos para abordagem do assunto $[20,23]$.

Como profissionais da saúde e pesquisadores, temos o papel de desmistificar tabus e construir saberes, proporcionando vida sexual saudável e digna para as idosas [5]. Estudos que explorem fatores envolvidos e dificuldades encontradas por essas clientes contribuem para o cuidado holístico. Desta forma, torna-se importante mais pesquisas sobre o tema, ainda tão pouco investigado, avançando no campo da geriatria/gerontologia e repercutindo no bem-estar e qualidade de vida $[11,16]$.

Os instrumentos como a Escala de Atitudes e Conhecimento Sobre Sexualidade no Envelhecimento (ASKAS) e a escala Quociente Sexual -Versão Feminina (QS-F) podem ser utilizados em pesquisas para análise da sexualidade de idosas, fornecendo subsídios para estratégias em saúde sexual.

ASKAS é uma escala de origem norte-americana com o objetivo de medir o conhecimento e atitudes acerca da sexualidade na velhice, idealizada por White (1982). Tem sido muito utilizada em estudos internacionais [24]. Foi traduzida, adaptada e validada para versão brasileira por Viana et al. (2008), contendo vinte questões na primeira parte (falso/verdadeiro/não sei) que medem o conhecimento, variação de 20 a 60 . Na segunda parte, oito questões (escala Likert de 5 pontos: discordo fortemente, discordo parcialmente, não concordo nem discordo, concordo parcialmente e concordo fortemente) que avaliam as atitudes, variação de 8 a 40 . Quanto menor o escore do entrevistado maior o conhecimento sobre sexualidade na velhice e atitude positiva em relação ao interesse do idoso por sexo. Pode ser aplicada em grupos ou individualmente, e assistida em casos de pesquisa com idosos de baixa escolaridade [25].

O QS-F foi desenvolvido no Programa de Estudos em Sexualidade (ProSex) do Instituto de Psiquiatria do Hospital das Clínicas da Faculdade de Medicina da Universidade de São Paulo. $\mathrm{O}$ instrumento compõe-se de 10 questões, devendo ser respondida numa escala de 0 a 5 . 0 resultado da soma das 10 respostas deve ser multiplicado por dois, o que resulta num índice total que varia de 0 a 100. Valores maiores indicam melhor desempenho/satisfação sexual: 82 a 100 pontos (bom a excelente); 62 a 80 (regular a bom); 42-60 (desfavorável a regular); 22 a 40 (ruim a desfavorável); 0 a 20 (nulo a ruim). É um instrumento útil para avaliar o desempenho e a satisfação sexual feminina, elaborado especialmente para população brasileira. Os domínios do QS-F avaliam todas as fases do ciclo de resposta sexual além de contemplar outros domínios relacionados à sexualidade feminina [26].

A satisfação sexual tem forte relação com a autoestima positiva, saúde mental e física, proporcionando melhor interação entre os parceiros [13].

\section{Saúde sexual e exame citopatológico}

O câncer de colo uterino é um problema de saúde pública com altas taxas de incidência e mortalidade. Estratégias de controle são necessárias através da promoção da saúde, prevenção, detecção precoce, tratamento e cuidados paliativos se necessário [27].

A detecção precoce é feita pelo rastreamento através do exame citopatológico (Papanicolau) [27]. O enfermeiro tem papel fundamental neste contexto, realiza a coleta do exame e propõe intervenções educativas direcionadas ao câncer de colo uterino, incentivando o autocuidado. Essas ações contribuem para a adesão e realização do exame pela idosa, pois algumas não possuem informações sobre a doença e a relevância do Papanicolau. Como não realizam o exame de forma rotineira, tornam-se vulneráveis $[28,29]$. No entanto, o cuidado de enfermagem direcionado para a sexualidade das idosas não deve se restringir apenas ao Papanicolau, mas necessita de cuidados específicos para o avançar da senescência [2,9].

Com o aumento de perspectiva da vitalidade e com as descobertas modernas que favorecem o desejo sexual, os idosos vêm evidenciando alterações nas atividades sexuais, no entanto desconhecem informações sobre sexo seguro, tornando vulneráveis a doenças sexualmente transmissíveis e ao Vírus da Imunodeficiência Humana (HIV). Algumas idosas acreditam que o preservativo não é necessário, pois não podem mais engravidar. Desta forma, apresentam comportamento de risco. É fundamental intervenções de políticas públicas com 
desenvolvimento de estratégias preventivas, diagnóstico e restauração da saúde frente a essas doenças [30].

\section{Consulta de enfermagem: abordando a sexualidade}

O processo de enfermagem é um instrumento metodológico, privativo do enfermeiro, orientando o cuidado de enfermagem e a documentação da prática profissional. Envolve cinco etapas: coleta de dados, diagnóstico de enfermagem, planejamento, implementação e avaliação de enfermagem. Quando realizado em instituições prestadoras de serviços ambulatoriais, domicílios, escolas e associações comunitárias é denominado de consulta de enfermagem [31].

As consultas de enfermagem devem ser um espaço confortável, com vínculo de confiança e respeito, considerando que cada idosa necessita de atenção personalizada e possui contextos diferentes [9]. Todas as áreas que são afetadas no envelhecimento, inclusive sexual, devem ser abordadas [32].

A consulta aborda assuntos íntimos e regiões que envolvem pudor, nesta oportunidade, deve aproveitar para abordagem de assuntos relacionados à sexualidade, ao meio psicossocial e possíveis disfunções sexual [11].

São barreiras para a sexualidade de idosas: perdas hormonais, mudanças corporais, doenças crônicas, algumas medicações e disfunções sexuais [16]. Como causas socioculturais temos viuvez, falta de privacidade e de informação, além disso, temos a influência da família, igreja e sociedade [13]. Nas emocionais incluem a ansiedade, depressão, culpa e vergonha [13]. Também temos que considerar que parceiros com impotência sexual, falta de desejo ou limitação física prejudicam a sexualidade das mesmas [8].

O enfermeiro tem como função orientar alternativas para intervir, atenuar e compensar os desgastes que afetam o desempenho da sexualidade das idosas [33]. Se não puder resolver, deverá encaminhar para o profissional pertinente [20]. A consulta de enfermagem auxiliará no compartilhamento do conhecimento, contribuindo com o enfrentamento da sexualidade no envelhecimento com naturalidade, permitindo sua vivência de forma livre e prazerosa [9].

É fundamental incluir a sexualidade de forma sistematizada nas rotinas do cuidado de enfermagem, contribuindo para minimização de tabus e preconceitos. No entanto, é necessário mudanças de paradigmas em saúde, na dinâmica do trabalho e reflexões sobre valores pessoais [9].

\section{Sensibilização}

A sensibilização sobre sexualidade no envelhecimento deve ser realizada com as idosas e com os profissionais de saúde. O enfermeiro tem papel fundamental nesse desafio, focando na importância da vivência sexual e seus benefícios, novas concepções, quebra de tabus e visão profissional ampliada. A sensibilização possibilita engajamento e reflexão sobre questões subjetivas $[8,34]$.

Falar sobre sexo causa desconforto, receio e pudor para as idosas que evitam expor suas experiências sexuais e possuem postura reservada e atitudes negativas sobre o tema [30].

A discussão de questões inerentes à sexualidade das idosas inclui um repensar da sociedade e de profissionais da saúde, para que possam ser abordadas sem reduzi-las aos aspectos biológicos, abrangendo percepções do corpo, prazer e valores emergentes sobre sexualidade na contemporaneidade [9].

A sexualidade está diretamente relacionada a percepção de qualidade de vida e, por ser uma função vital humana, interfere no desempenho social, profissional, físico e psíquico [11].

\section{Apoio e acolhimento}

Acolher significa receber pessoas e a forma desse acolhimento vai ser determinante para o tratamento e para não perder o sujeito na continuidade [35].

É necessário acolher as idosas compreendendo e auxiliando nas suas dificuldades, valorizando a ligação entre medidas preventivas e assistenciais. Estratégias que proporcionem conforto na abordagem da sexualidade devem ser utilizadas, ganhando credibilidade dessa população [9]. 
As modificações corporais decorrentes do envelhecimento geram angústias e dúvidas, cabendo aos profissionais apoiar emocionalmente, oferecendo suporte e alternativas para amenizar essas mudanças [36].

A enfermagem tem nas mãos ferramentas fundamentais para lidar com esse declínio. É importante possuir habilidades para atender as diversidades vivenciadas por cada pessoa [9].

Os enfermeiros podem trabalhar a temática sexualidade numa perpectiva crítica, utilizando oficinas para construção de autonomia e emancipação da liberdade sexual. Rodas de conversas com escuta qualificada entre idosas podem ser um momento para trocas dialógicas referentes aos anseios, dúvidas, curiosidades e angústias sobre sexualidade. Desta forma, podemos apoiar e acolher essa população [1].

Conclusão

As evidências encontradas neste estudo demonstram a complexidade do tema. A enfermagem pode contribuir de vários modos e nos diversos espaços do cuidar, pois estão interligados e se complementando. Ressalta-se a importância desse profissional, considerando a categoria mais presente com as idosas. No entanto, deve ser preparado para a abordagem da sexualidade no envelhecimento, compreendendo as particularidades e contextos diferentes. É imprescindível um novo olhar para esse assunto, através da educação em saúde tanto para as idosas quanto para profissionais, em busca do cuidado holístico, vida sexual saudável e satisfatória, livre de preconceitos e violência.

Desse modo, existe um desafio para o enfermeiro, pois terá que mudar também suas concepções sobre sexualidade na terceira idade, tratar a questão com naturalidade. Novas investigações são necessárias para enriquecer a compreensão e a prática clínica sobre o tema.

\section{Referências}

1. Oliveira EL, Neves ALM, Silva IR. Sentidos de sexualidade entre mulheres idosas: relações de gênero, ideologias mecanicistas e subversão. Psicol Soc 2018;30:e166019. https://doi.org/10.1590/1807-0310/2018v30166019

2. Cabral NES, Lima CFM, Rivemales MCC, Souza US, Silva BMC. Compreensão da sexualidade por idosas de área rural. Rev Bras Enferm 2019;72(Supl 2):147-52. https://doi.org/10.1590/0034-7167-2018-0385

3. Uchôa YS, Costa DCA, Silva Junior IAP, Silva STSE, Freitas WMTM, Soares SCS. A sexualidade sob o olhar da pessoa idosa. Rev Bras Geriatr Gerontol 2016;19(6):93949. https://doi.org/10.1590/1981-22562016019.150189

4. Vieira KFL, Nóbrega RPM, Arruda MVS, Veiga PMM. Representação social das relações sexuais: um estudo transgeracional entre mulheres. Psicol Ciênc Prof 2016;36(2):329-40. https://doi.org/10.1590/1982-3703001752013

5. Souza M, Marcon SS, Bueno SMV, Carreira L, Baldissera VDA. A vivência da sexualidade por idosas viúvas e suas percepções quanto à opinião dos familiares a respeito. Saúde Soc 2015;24(3):936-44. https://doi.org/10.1590/S010412902015132060

6. Sehnem G, Schmalfuss J, Bonadiman P, Pereira F, Lipinski J, Bogorni L. Gênero e sexualidade: influências na prevenção das DSTs/AIDS e as contribuições para a enfermagem. Rev Enferm UFSM 2015;4(4):678-88.

https://doi.org/10.5902/2179769212408

7. Vieira KFL, Coutinho MPL, Saraiva ERA. A Sexualidade na velhice: representações sociais de idosos frequentadores de um grupo de convivência. Psicol Ciênc Prof 2016;36(1):196-209. https://doi.org/10.1590/1982-3703002392013

8. Santos AD, Santos ALS, Andrade LM, Boa Sorte ET, Santos ES, Guerra SS. Concepção de mulheres idosas sobre a sexualidade na velhice. Rev Enferm UFPE 2019;13:e241752. https://doi.org/10.5205/1981-8963.2019.241752

9. Souza CL, Gomes VS, Silva RL, Silva ES, Alves JP, Santos NR et al. Envelhecimento, sexualidade e cuidados de enfermagem: o olhar da mulher idosa. Rev Bras Enferm 2019;72(Supl 2):71-8. https://doi.org/10.1590/0034-7167-2018-0015

10. Alencar RA, Ciosak SI. Aids em idosos: motivos que levam ao diagnóstico tardio. Rev Bras Enferm 2016;69(6):1140-46. https://doi.org/10.1590/0034-7167-2016-0370 
11. Rodrigues LR, Portilho P, Tieppo A, Chambo Filho A. Análise do comportamento sexual de idosas atendidas em um ambulatório de ginecologia. Rev Bras Geriatr Gerontol 2018;21(6):724-30. https://doi.org/10.1590/1981-22562018021.180090

12. Rother ET. Revisão sistemática X revisão narrativa. Acta Paul Enferm 2007;20(2):5-6. Disponível em: https://www.scielo.br/pdf/ape/v20n2/a01v20n2.pdf

13. Nascimento R, Marin M, Pirolo S, Lacerda M. Vivência da sexualidade por mulheres idosas. Rev Enferm UERJ 2017;25:e20892. https://doi.org/10.12957/reuerj.2017.20892

14. Rodrigues DMMR, Nogueira IS, Higarashi IH, Heidemann ITSB, Baldissera VDA. Desvelamento crítico em sexualidade entre idosas como dispositivo de avaliação educativa dialógica. Rev Baiana Enferm 2019;33:e27754. https://doi.org/10.18471/rbe.v33.27754

15. Rodrigues DMMR, Labegalini CMG, Higarashi IH, Heidemann ITSB, Baldissera VDA. O percurso educativo dialógico como estratégia de cuidado em sexualidade com idosas. Esc Anna Nery 2018;22(3):e20170388. https://doi.org/10.1590/2177-9465-ean-2017$\underline{0388}$

16. Crema IL, Tilio R, Campos MTA. Repercussões da menopausa para a sexualidade de idosas: revisão integrativa da literatura. Psicol Ciênc Prof 2017;37(3):753-69. https://doi.org/10.1590/1982-3703003422016

17. Nakata PT, Costa FM, Bruzamolin CD. Cuidados de enfermagem ao idoso na Estratégia de Saúde da Família: revisão integrativa. Rev Enferm UFPE 2017;11(supl1):393-402. https://doi.org/10.5205/1981-8963-v11i1a11920p393-4022017

18. Goes T, Polaro S, Gonçalves L. Cultivo do bem viver das pessoas idosas e tecnologia cuidativo-educacional de enfermagem. Enferm Foco 2016;7(2):47-51. https://doi.org/10.21675/2357-707X.2016.v7.n2.794

19. Freire P. Pedagogia da autonomia: saberes necessários à prática educativa. 55ed. São Paulo: Paz e Terra; 2017.

20. Muliira JK, Muliira RS. Sexual health for older women: implications for nurses and other healthcare providers. Sultan Qaboos Univ Med J 2013;13(4):469-76. https://doi.org/10.12816/0003304

21. Venturini L, Beuter M, Leite MT, Bruinsma JL Backes C. Atuação da equipe de enfermagem frente à sexualidade de idosas institucionalizadas. Rev Esc Enferm USP 2018;52:e03302. https://doi.org/10.1590/s1980-220x2017017903302

22. Freitas CM, Freitas CASL, Parente JRF, Vasconcelos MIO, Lima GK, Mesquita KO, Martins SC, Mendes JDR. Uso de metodologias ativas de aprendizagem para a educação na saúde: análise da produção científica. Trab Educ Saúde 2015;13(Supl2):117-30. https://doi.org/10.1590/1981-7746-sip00081

23. Souza CA, Cardoso FL, Silveira RA, Wittkopf PG. Comportamento da frequência cardíaca em adulto jovem durante exercício físico e atividade sexual. Rev Bras Med Esporte 2012;18(5):345-6. https://doi.org/10.1590/S1517-86922012000500013

24. Charles BW. A scale for the assessment of attitudes and knowledge regarding sexuality in the aged. Arch Sex Behav 1982;11(6):491-502. Disponível em: https://link.springer.com/article/10.1007/BF01542474

25. Viana HB. Adaptação e validação da escala ASKAS: Aging Sexual Knowledge and Atitudes Scale em idosos brasileiros [Internet] [tese]. Campinas, SP: Universidade Estadual de Campinas; 2008 [citado em 24 ago 2020]. Disponível em: http://repositorio.unicamp.br/bitstream/REPOSIP/275175/1/Vi.ana HelenaBrandao D.p df

26. Abdo CHN. Quociente sexual feminino: um questionário brasileiro para avaliara atividade sexual da mulher. Diagn Tratamento 2009;14(2):89-90. Disponível em: http://files.bvs.br/upload/S/1413-9979/2009/v14n2/a0013.pdf

27. Ministério da Saúde (Brasil), Secretaria de Atenção à Saúde, Departamento de Atenção Básica. Controle dos cânceres do colo do útero e da mama. Brasília, DF: Ministério da Saúde; 2013.

28. Fonseca Neto AA, Pereira Filho AM, Silva AB. Conhecimento de idosas de uma unidade da estratégia saúde da família sobre prevenção do câncer cervicouterino. Braz J Health Rev 2020;3(2):1699-712. https://doi.org/10.34119/bjhrv3n2-032

29. Abreu FA, Santo FHE, Chibante CLP, Santos TD, Brito WAP. Prevenção do câncer de mama e cérvico-uterino em idosas de um grupo de convivência. Rev Enferm Atual In 
Derme 2019;78(16):29-34. Disponível em:

https://revistaenfermagematual.com.br/index.php/revista/article/view/357/240

30. Aguiar RB, Leal MCC, Marques APO. Conhecimento e atitudes sobre sexualidade em pessoas idosas com HIV. Ciênc Saúde Colet 2020;25(6):2051-62. https://doi.org/10.1590/1413-81232020256.18432018

31. Conselho Federal de Enfermagem (Brasil). Resolução COFEN 358 / 2009. Dispõe sobre a Sistematização da Assistência de Enfermagem e a implementação do Processo de Enfermagem em ambientes, públicos ou privados, em que ocorre o cuidado profissional de Enfermagem, e dá outras providências [Internet]. Brasília, DF: COFEN; 2009 [citado 2020 Mar 23]. Disponível em: http://www.cofen.gov.br/resoluocofen-3582009 4384.html

32. Valcarenghi RV, Lourenço LFL, Siewert JS, Alvarez AM. Produção científica da enfermagem sobre promoção de saúde, condição crônica e envelhecimento. Rev Bras Enferm 2015;68(4):705-12. https://doi.org/10.1590/0034-7167.2015680419i

33. Ruoco MTM, Brêtas ACP, Figueiredo EM. Quem falou que idosa só fica em casa? Rev Enferm UERJ 2014;22(5):693-98. https://doi.org/10.12957/reueri.2014.4913

34. Dutra SG, Rubim PEN, Denardin Budó ML, Machado da Silva F, Ressel LB. A construção da sexualidade de estudantes de enfermagem e suas percepções acerca da temática. Cienc Enferm 2014;20(1):111-21. https://doi.org/10.4067/S071795532014000100010

35. Silva SMG, Silva FWF. Acolhimento e atendimento humanizado em saúde mental: uma revisão integrativa [Internet] [trabalho de conclusão de curso]. São Francisco do Conde, BA: Universidade da Integração Internacional da Lusofonia Afro-Brasileira; 2020 [citado em 24 ago 2020]. 14 f. Disponível em: http://repositorio.unilab.edu.br:8080/jspui/bitstream/123456789/1884/1/2020 arti silvina silva.pdf

36. Calegari RC, Massarollo MCKB, Santos MJ. Humanização da assistência à saúde na percepção de enfermeiros e médicos de um hospital privado. Rev Esc Enferm USP 2015;49(spe2):42-47. https://doi.org/10.1590/S0080-623420150000800006 